\title{
A Case of Morvan Syndrome Converted from Isaacs' Syndrome after Thymectomy with Positivity for Both Anti-LGI1 and Anti-CASPR2 Antibodies
}

\author{
Daisuke Suzuki ${ }^{1}$, Yoshihiro Suzuki ${ }^{1}$, Daisuke Sato ${ }^{1}$, Kenji Kikuchi ${ }^{1}$, Naoki Kanauchi ${ }^{2}$, \\ Akiko Nishida $^{3}$ and Yasuyuki Ohta ${ }^{4}$
}

\begin{abstract}
:
Anti-voltage-gated potassium channel complex antibodies-mediated disorder includes Isaacs' syndrome, which is characterized by neuromyotonia, and Morvan syndrome, which is characterized by neuromyotonia, encephalopathy and autonomic dysfunction. We herein report a patient with Morvan syndrome that converted from Isaacs' syndrome after thymectomy. The patient first presented with myospasm in all extremities and positivity for both anti-leucine-rich glioma inactivated 1 (LGI1) and anti-contactin-associated protein like 2 (CASPR2) antibodies and subsequently developed encephalopathy after thymectomy, which was successfully improved by immunotherapy. This is the first case of Morvan syndrome wherein thymectomy worsened Isaacs' syndrome, suggesting that immunotherapy should be considered for Isaacs' syndrome accompanied by positivity for both anti-LGI1 and anti-CASPR2 antibodies to prevent worsening to Morvan syndrome.
\end{abstract}

Key words: CASPR2, Isaacs' syndrome, LGI1, Morvan syndrome, thymectomy

(Intern Med Advance Publication)

(DOI: 10.2169/internalmedicine.8145-21)

\section{Introduction}

Anti-voltage-gated potassium channel (VGKC) complex antibodies are associated with various neurological symptoms involving both the peripheral and central nervous systems (1). Isaacs' syndrome is characterized by neuromyotonia due to peripheral nerve hyperexcitability, while Morvan syndrome is a complex neurological disorder including neuromyotonia, encephalopathy and autonomic dysfunction. Anti-VGKC complex antibodies-mediated disorder is often associated with thymoma (1), and the disease sometimes develops after thymectomy $(2,3)$. However, there have been no reports of thymectomy causing Isaacs' syndrome to worsen to Morvan syndrome.

We herein report the first case of Morvan syndrome converted from Isaacs' syndrome after thymectomy.

\section{Case report}

A 49-year-old Japanese man had mild myospasm and pain in all extremities when he was found to have an anterior mediastinal tumor by chest computed tomography (CT) (FigureA). He was diagnosed with stage IIB thymoma according to the Masaoka staging system, and thymothymectomy combined with resection of the mediastinal pleura and pericardium was performed in our hospital the next month, revealing a type B2 thymoma pathologically according to the World Health Organization criteria (FigureB). Five months after thymectomy, his myospasm and pain worsened, and he showed body weight loss (15 kg loss over 6 months) and insomnia caused by myospasm. Thus, he was referred to our hospital.

A neurological examination found fasciculation and myokymia on the face and all extremities and normal eye move-

\footnotetext{
${ }^{1}$ Department of Neurology, Nihonkai General Hospital, Japan, ${ }^{2}$ Department of Respiratory Surgery, Nihonkai General Hospital, Japan, ${ }^{3}$ Department of Pathology, Nihonkai General Hospital, Japan and ${ }^{4}$ Division of Neurology and Clinical Neuroscience, Department of Internal Medicine III, Yamagata University Faculty of Medicine, Japan

Received: June 16, 2021; Accepted: September 6, 2021; Advance Publication by J-STAGE: October 19, 2021

Correspondence to Dr. Yasuyuki Ohta, yasuyuki@med.id.yamagata-u.ac.jp
} 
A

B
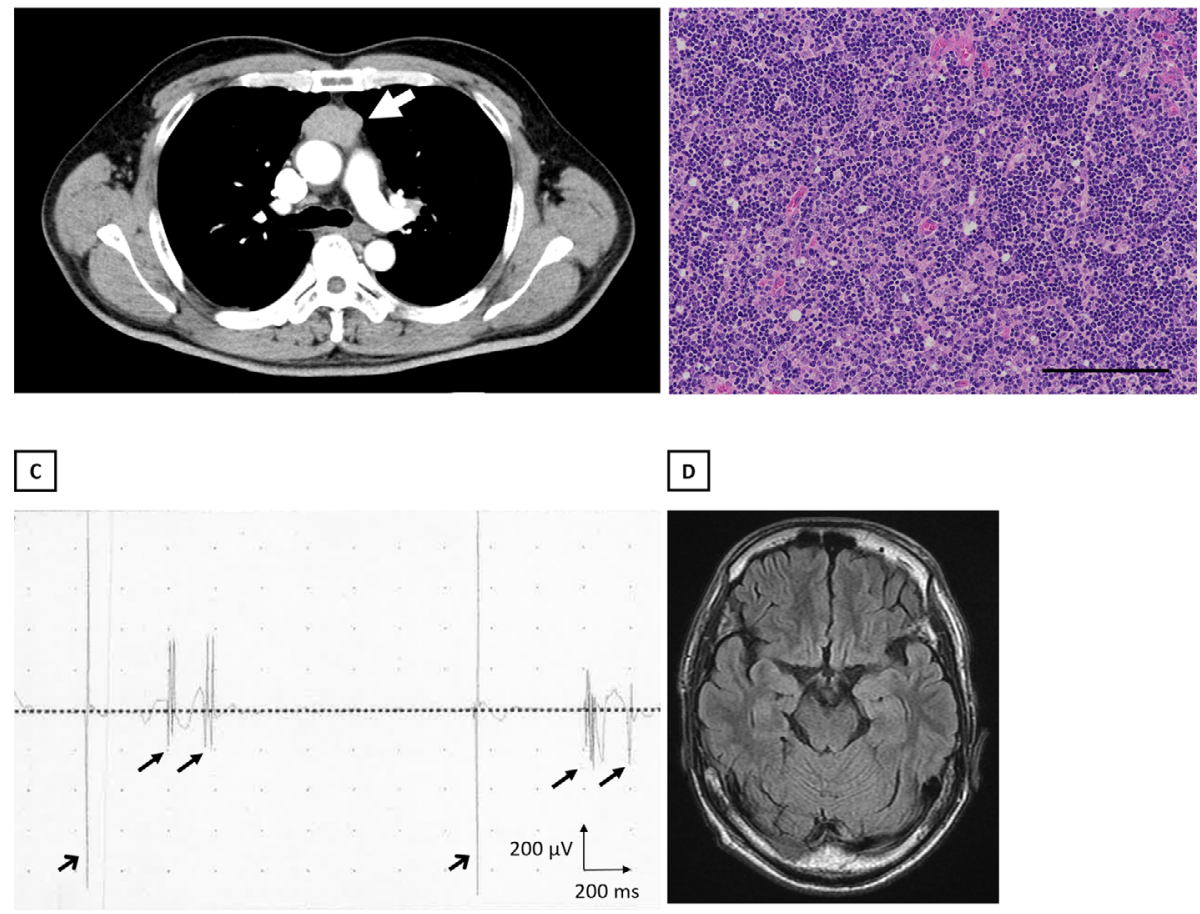

D

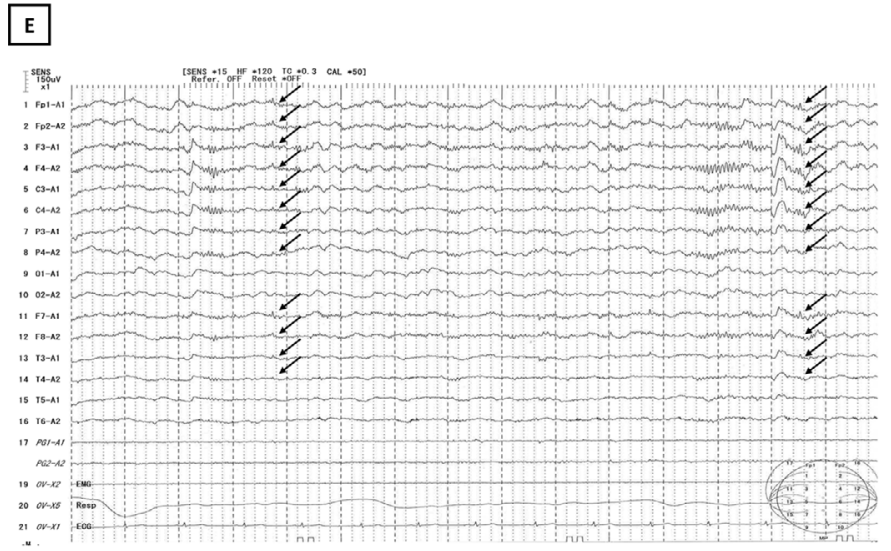

Figure. Chest contrast-enhanced CT showing a thymoma (A, arrow). Histopathological findings of thymoma showing abundant lymphocytes and neoplastic epithelial cells (B, scale bar; $200 \mu \mathrm{m})$. An EMG study at the right rectus femoris showing myokymic discharges and fasciculations $(\mathrm{C}$, small and large arrows respectively, scale bar; vertical axis: $200 \mu \mathrm{V}$, horizontal axis: $200 \mathrm{~ms}$ ). Brain MRI showing normal findings on FLAIR (D). An EEG showing generalized spikes that were left frontaldominant (E, arrows).

ment without blepharoptosis. He had pain in all extremities but showed no muscle atrophy, weakness or easy fatigability, accompanied by normoreflexia, and normal superficial and deep senses as well as cerebellar and autonomic systems. He also showed mild sleep disturbance and anxiety but no memory disturbance.

Serum analyses showed no inflammatory reaction but did reveal positivity for anti-acetylcholine receptor (AchR) antibody $(1.1 \mathrm{nmol} / \mathrm{L}$, normal $<0.2 \mathrm{nmol} / \mathrm{L})$. Both anti-leucinerich glioma inactivated 1 (LGI1) and anti-contactinassociated protein like 2 (CASPR2) antibodies, subtypes of voltage-gated potassium channel (VGKC) complex, were also positive on an indirect immunofluorescence assay (Cos- mic Corporation, Tokyo, Japan). The tensilon test did not improve the frequency of myospasm. Whole-body CT and fluorodeoxyglucose positron emission tomography suggested no inflammatory or malignant lesions. Electromyography (EMG) revealed myokymic discharges in the form of doublets and multiplets as well as fasciculations in all extremities (FigureC). Since his dominant symptoms were peripheral nerve symptoms, he was diagnosed with Isaacs' syndrome. He was started on treatment with carbamazepine (CBZ), but he stopped taking CBZ after a few days because of dizziness as a side effect.

Two months after the diagnosis of Isaacs' syndrome, he was admitted to our hospital with tonic-clonic seizure, delir- 
ium, hallucination, agitation and insomnia. He still showed fasciculation, myokymia and pain in all extremities. An examination of the cerebral spinal fluid showed a normal cell count and protein. Brain magnetic resonance imaging (MRI) showed normal findings on fluid-attenuated inversion recovery (FLAIR) (FigureD). However, electroencephalography (EEG) showed generalized spikes that were left frontaldominant (FigureE). Therefore, he was diagnosed with Morvan syndrome that had converted from Isaacs' syndrome.

After treatment with two courses of methylprednisolone pulse (mPSL) and plasma exchange, followed by oral prednisolone, all of his symptoms gradually improved, and he was discharged 32 days after the start of treatments.

\section{Discussion}

Anti-VGKC complex antibody-mediated disorder shows a wide spectrum of neurological symptoms involving the peripheral and central nervous systems, including Isaacs' syndrome, which is characterized by peripheral nerve hyperexcitability, and Morvan syndrome, which is recognized as a combination of peripheral nerve hyperexcitability, encephalopathy and autonomic failures (1). Anti-LGI1 antibody is reportedly associated with delusion, mood change and myoclonus, while anti-CASPR2 antibody is associated with tumors and body weight loss (1). However, both anti-LGI1 and anti-CASPR2 antibodies are sometimes positive in cases with Isaacs' or Morvan syndrome (1).

The present patient initially showed mild myospasm and pain in all extremities when thymectomy was performed, which progressed to moderate peripheral nervous symptoms of fasciculation, myokymia and pain in the face and all extremities accompanied by positivity for both anti-LGI1 and anti-CASPR2 antibodies. A total of $6.9 \%$ of Isaacs' syndrome patients with positive anti-VGKC complex antibody were reported to show insomnia (1), and the present patient presented with insomnia caused by myotonia, although no severe insomnia with mental confusion, hallucinations or agitation, a phenomenon known as 'agrypnia excitata' and characteristic of Morvan syndrome. Thus, he was first diagnosed with Isaacs' syndrome. However, two months after this diagnosis, he mainly showed hallucination, agitation and tonic-clonic seizure as encephalopathy. Therefore, he was finally diagnosed with Morvan syndrome that had converted from Isaacs' syndrome, and was successfully treated by im- munotherapy, including mPSL and plasma exchange, as in previous reports (4).

Anti-VGKC complex antibody-mediated disorder is sometimes associated with neoplasms, such as thymoma (1). The disease often develops several years before the discovery of the neoplasm but can occasionally occur a few weeks after surgery, such as thymectomy $(2,3)$. Since thymectomy for Morvan syndrome with thymoma sometimes worsens the symptoms (3), whether or not thymectomy should be performed for Morvan syndrome is controversial. However, immunotherapy, such as mPSL and plasma exchange, are effective against Isaacs' syndrome or Morvan syndrome accompanied by both anti-LGI1 and CASPR2 antibodies. If immunotherapy had been performed for the present patient in the early stage, when he showed only peripheral nervous symptoms, the progression to Morvan syndrome might have been prevented.

To our knowledge, the present patient is the first case of Morvan syndrome converted from Isaacs' syndrome after thymectomy. These findings suggest that thymectomy might cause Isaacs' syndrome to worsen to Morvan syndrome in patients positive for both anti-LGI1 and anti-CASPR2 antibodies. Immunotherapy should be considered for cases of Isaacs' syndrome accompanied by positivity for both antiLGI1 and CASPR2 antibodies in order to prevent worsening to Morvan syndrome.

The authors state that they have no Conflict of Interest (COI).

\section{References}

1. Irani SR, Pettingill P, Kleopa KA, et al. Morvan syndrome: clinical and serological observations in 29 cases. Ann Neurol 72: 241255, 2012.

2. Fleisher J, Richie M, Price R, et al. Acquired neuromyotonia heralding recurrent thymoma in myasthenia gravis. JAMA Neurol 70: 1311-1314, 2013.

3. Cottrell DA, Blackmore KJ, Fawcett PR, et al. Sub-acute presentation of Morvan's syndrome after thymectomy. J Neurol Neurosurg Psychiatry 75: 1504-1505, 2004.

4. Maskery M, Chhetri SK, Dayanandan R, et al. Morvan syndrome: a case report with patient narrative and video. Neurohospitalist 6 : 32-35, 2016.

The Internal Medicine is an Open Access journal distributed under the Creative Commons Attribution-NonCommercial-NoDerivatives 4.0 International License. To view the details of this license, please visit (https://creativecommons.org/licenses/ by-nc-nd/4.0/).

\section{(C) The Japanese Society of Internal Medicine} Intern Med Advance Publication 